\title{
Effect of the Yield Criterion of Matrix on the Brittle Fracture of Fibres in Uniaxial Tension of Composites
}

\author{
Sergei Alexandrov, ${ }^{1}$ Yaroslav Erisov, ${ }^{2}$ and Fedor Grechnikov ${ }^{2}$ \\ ${ }^{1}$ Institute for Problems in Mechanics, Russian Academy of Sciences, 101-1 Prospect Vernadskogo, Moscow 119526, Russia \\ ${ }^{2}$ Samara State Aerospace University, 34 Moskovskoe Shosse, Samara 443086, Russia \\ Correspondence should be addressed to Yaroslav Erisov; yaroslav.erisov@mail.ru
}

Received 22 March 2016; Revised 12 April 2016; Accepted 13 April 2016

Academic Editor: Filippo Berto

Copyright (c) 2016 Sergei Alexandrov et al. This is an open access article distributed under the Creative Commons Attribution License, which permits unrestricted use, distribution, and reproduction in any medium, provided the original work is properly cited.

\begin{abstract}
This paper examines the effect of the yield criterion of the matrix on brittle fracture of the fibre in continuous fibre reinforced metal matrix composites subjected to tension in the direction parallel to the fibres. It is assumed that the matrix obeys quite a general isotropic yield criterion. An approximate approach to predicting the tensile load at which the fibre breaks previously proposed in the literature is adopted. It is shown that this tensile load is practically independent of the yield criterion of the matrix. This is a great advantage for engineering applications since an analytic solution is available in the case of Tresca yield criterion. This solution can be used for a wide range of matrix materials with no loss of the accuracy of the prediction of the tensile load at which the fibre breaks.
\end{abstract}

\section{Introduction}

There are many applications in which continuous and short fibre reinforced metal matrix composites are required. There is a class of such composites in which the fibre is made of brittle material and the matrix of ductile material [1-3]. Failure of such composites involves various mechanisms such as fibre fracture, interfacial debonding, and matrix plasticity. The effect of short fibre reinforcement on the fracture toughness of metal matrix composites has been studied in $[4,5]$. Interfacial debonding has been investigated in [68]. Computational methodologies for modeling fracture in continuous fibre reinforced metal matrix composites and in laminated composites at the micromechanical level have been proposed in [7-10]. It has been shown in [11] that the interfacial conditions strongly affect tensile fracture characteristics of a boron-fibre-reinforced aluminum composite.

In the present paper the brittle fracture of fibres in continuous fibre reinforced metal matrix composites subjected to uniaxial tension is predicted using the approach proposed in [1]. In the latter work, this approach has led to a simple analytic solution for the axial force at which the fibre breaks. In particular, the solution predicts the influence of the volume fraction of fibres on the strength of composites, which is well known from experiment [12]. Tresca's yield criterion has been adopted for the matrix in [1]. However, it is known that the strength of metal matrix composites is influenced by many factors including matrix strength [13]. Moreover, the in situ flow properties of the matrix of metal matrix composites differ from the properties of the matrix bulk material and the experimental scatter is much larger for experiments in the matrix than in the corresponding bulk material [14]. It is therefore of importance to extend the approach proposed in [1] to generalized yield criteria. Such criteria have been proposed, for example, in $[15,16]$. In the present paper, the criterion proposed in [15] is used. It is shown that the predicted magnitude of the axial force at which the fibre breaks is almost independent of the yield criterion of the matrix. Therefore, the analytical expression derived in [1] can be used for a large class of continuous fibre reinforced metal matrix composites. The main result of the present paper can be used in conjunction with methods developed to study the behaviour of composites in the presence of fractured fibres (e.g., [17]).

The rigid plastic solution found in the present paper is of academic interest as well. To the best of authors' knowledge, 


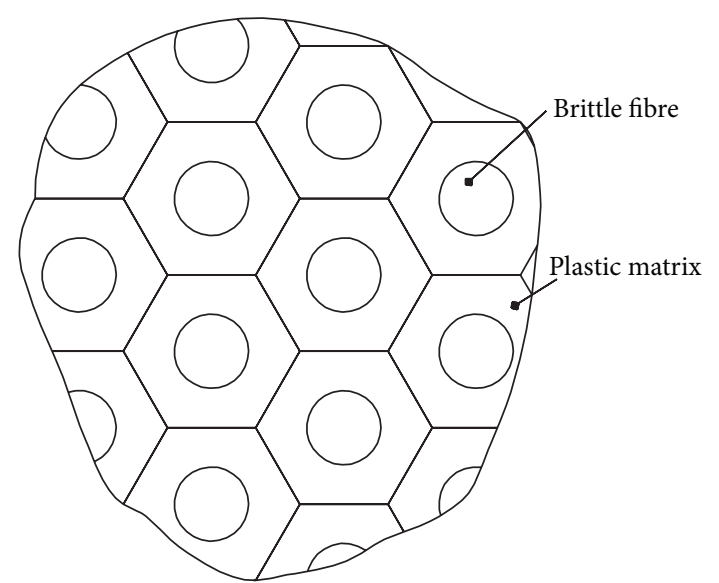

FIgURE 1: Typical cross section of the idealized composite.

the only available nontrivial axisymmetric semianalytic rigid plastic solution for the generalized yield criterion has been outlined in [18]. In this paper, flow through an infinite conical channel has been analyzed. An alternative solution to this problem and quantitative results have been presented in [19], where the yield criterion proposed in [15] was adopted. The present paper provides another solution. It is also worthy to note that the solution given in [1] has been extended to the double shearing model in [20] and to a viscoplastic model in [21]. The fracture of fibres has not been considered in these works. A description of the double shearing model can be found in [22].

\section{General Approach}

An elegant theory of the failure of ductile materials reinforced by elastic fibres has been proposed in [1]. This theory is outlined in this section to formulate the boundary value problem to be solved. It is assumed that the composite consists of a large number of equal cells, each of which is a hexagonal cylinder of the matrix material containing a concentric circular fibre. A typical cross section of the idealized composite is illustrated in Figure 1. These cells are further idealized replacing the hexagonal cylinders with circular cylinders of the same cross-sectional area. Figure 2 illustrates the cross section of a typical cell that will be used in the mathematical formulation. In particular, the radius of the fibre is denoted by $a_{0}$ and the radius of the cell by $b_{0}$. The length of the cell will be denoted by $2 L$.

It is supposed that the composite is subjected to uniaxial tension applied in the direction parallel to the fibres. The equations for the cell shown in Figure 2 are referred to a cylindrical polar coordinate system $(r, \theta, z)$ whose origin is located at the centre of the cell and $z$-axis lies along the axis of the fibre (Figure 3 ). The plane $z=0$ coincides with the plane of symmetry of the cell. It is assumed that the state of stress is approximately axially symmetric about the $z$-axis. Therefore, the nonzero stress components referred to the cylindrical coordinate system are $\sigma_{r r}, \sigma_{\theta \theta}, \sigma_{z z}$, and $\sigma_{r z}$. The material of the matrix is isotropic. Therefore, the nonzero strain rate

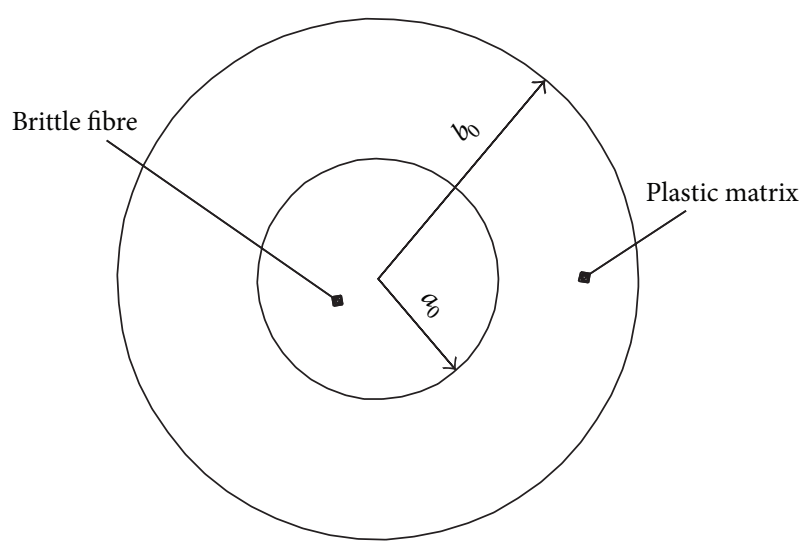

FIGURE 2: Cross section of a typical idealized cell.

components referred to the cylindrical coordinate system are $\xi_{r r}, \xi_{\theta \theta}, \xi_{z z}$, and $\xi_{r z}$. Moreover, the circumferential velocity vanishes everywhere. The radial and axial velocities will be denoted by $u_{r}$ and $u_{z}$, respectively.

Since the boundary value problem is symmetric about the plane $z=0$, it is sufficient to consider the region $z \geq 0$. Based on the assumptions made the following boundary conditions have been formulated in [1]:

$$
u_{r}=-U
$$

for $r=b_{0}$,

$$
u_{r}=0
$$

for $r=a_{0}$,

$$
u_{z}=0
$$

for $z=0$,

$$
\sigma_{r z}=0
$$

for $r=b_{0}$,

$$
\sigma_{r z}=\tau_{f}
$$

for $r=a_{0}$, and

$$
\left.\int_{0}^{L} \sigma_{r r}\right|_{r=b_{0}} d z=0 .
$$

In (5), $\tau_{f}$ is the shear yield stress of the matrix. The boundary conditions (1) to (6) should be used to solve the plasticity boundary value problem in the matrix. It is also assumed that the ends of the fibres do not support tensile load (Figure 3). This load is transferred to the fibre only through the shear stress on the contact surface. The resulting force acting in the axial direction at the cross section $z=L$ is

$$
P=\left.2 \pi \int_{a_{0}}^{b_{0}} \sigma_{z z}\right|_{z=L} r d r .
$$




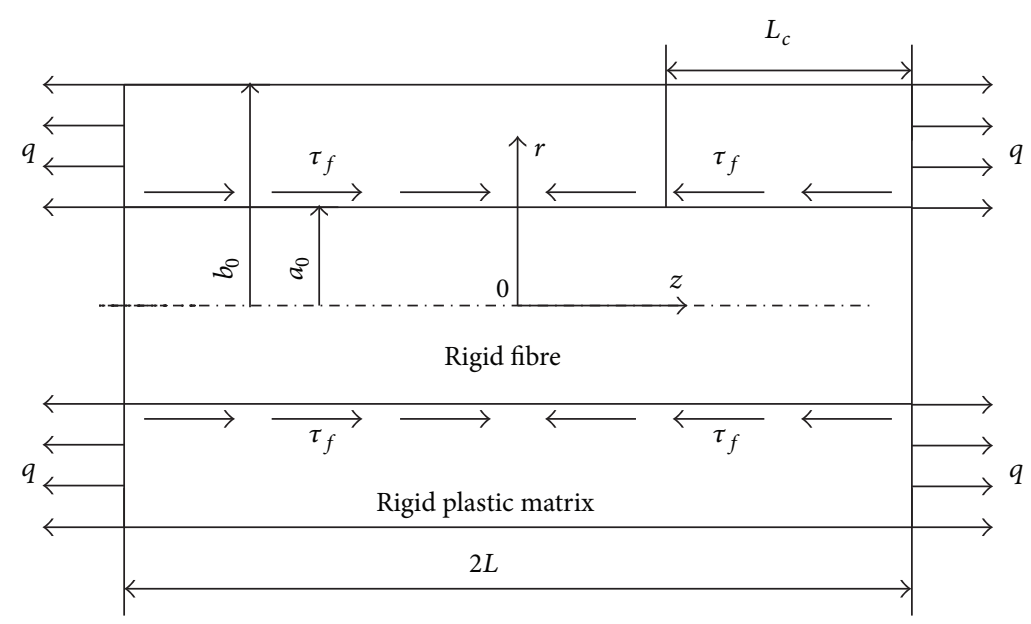

Figure 3: Configuration and coordinate system for a typical cell.

Let $T$ be the mean tensile stress at which the fibre breaks. It has been shown in [1] that failure occurs by plastic flow of the matrix if $L<L_{c}$ and by fracture of the fibre if $L>L_{c}$ where

$$
L_{c}=\frac{a_{0} T}{2 \tau_{f}}
$$

The approach to determining the tensile load at which the fibre breaks is as follows. It is assumed that in the matrix

$$
\begin{aligned}
& \frac{\sigma_{z z}}{\sigma_{0}}=1+C, \\
& \frac{\sigma_{r r}}{\sigma_{0}}=\frac{\sigma_{\theta \theta}}{\sigma_{0}}=C, \\
& \sigma_{r z}=0
\end{aligned}
$$

in the range $0 \leq z \leq L-L_{c}$ (Figure 3). Here $\sigma_{0}$ is the yield stress in tension and $C$ is an arbitrary constant. It is evident that the state of stress given by (9) satisfies any yield criterion. The state of stress in the matrix in the range $L-L_{c} \leq z \leq L$ should be found from the solution satisfying the boundary conditions (1) to (5). It is then assumed that

$$
\left[\int_{a_{0}}^{b_{0}} \sigma_{z z} r d r\right]=0
$$

at $L=L_{c}$. Here [ ] denotes the amount of jump in the quantity enclosed in the brackets. Equations (6) and (10) allow the axial stress in the matrix to be determined. Then, (7) can be used to find the tensile load at which the fibre breaks.

The approach outlined in this section has been adopted in [1] in conjunction with Tresca's yield criterion in the matrix. As a result, the dimensionless tensile load at which the fibre breaks has been found as

$$
\begin{aligned}
\frac{P}{\pi \sigma_{0} b_{0}^{2}} & =q_{S} \\
& =\left(1-a^{2}\right)\left[1-\frac{(2-n)}{2} \frac{L_{c}}{L}\right]+\frac{T a^{2}}{\sigma_{0}}\left(1-\frac{L_{c}}{2 L}\right),
\end{aligned}
$$

where $a=a_{0} / b_{0}$ and $n$ is a numerical coefficient. This coefficient is given by

$$
\begin{aligned}
n= & -\frac{1}{2}+\frac{a^{2}}{\left(1-a^{2}\right)} \ln a-\frac{\left(1-a^{2}\right)}{a^{2}} \\
& -\frac{\left(1+a^{2}\right)}{\left(1-a^{2}\right)}\left[a^{2} F(\gamma, \phi)-\frac{E(\gamma, \phi)}{a^{2}}\right] .
\end{aligned}
$$

Here $F(\gamma, \phi)$ and $E(\gamma, \phi)$ are the incomplete elliptic integrals of the first and second kinds, respectively, and

$$
\begin{aligned}
& \gamma=\sqrt{1-a^{4}}, \\
& \phi=\arctan \left(\frac{1}{a}\right) .
\end{aligned}
$$

Many materials satisfy other yield criteria compared to that of Tresca. Therefore, it is of interest to correct (11) taking into account a generalized yield criterion or to show that this simple equation is accurate enough for predicting the tensile load at which the fibres breaks. In the present paper, the yield criterion proposed in [15] is adopted.

\section{Rigid Plastic Solution in the Matrix}

Let $\sigma_{1}, \sigma_{2}$, and $\sigma_{3}$ be the principal stresses. With no loss of generality it is possible to assume that

$$
\sigma_{1} \geq \sigma_{2} \geq \sigma_{3} .
$$

Then, the yield criterion proposed in [15] can be written as

$$
\left[\frac{\left(\sigma_{1}-\sigma_{2}\right)^{m}+\left(\sigma_{2}-\sigma_{3}\right)^{m}+\left(\sigma_{1}-\sigma_{3}\right)^{m}}{2}\right]^{1 / m}=\sigma_{0} .
$$

The associated flow rule is

$$
\begin{aligned}
& \xi_{1}=\lambda\left[\left(\sigma_{1}-\sigma_{2}\right)^{m-1}+\left(\sigma_{1}-\sigma_{3}\right)^{m-1}\right], \\
& \xi_{2}=\lambda\left[\left(\sigma_{2}-\sigma_{3}\right)^{m-1}-\left(\sigma_{1}-\sigma_{2}\right)^{m-1}\right], \\
& \xi_{3}=-\lambda\left[\left(\sigma_{2}-\sigma_{3}\right)^{m-1}+\left(\sigma_{1}-\sigma_{3}\right)^{m-1}\right],
\end{aligned}
$$


where $\xi_{1}, \xi_{2}$, and $\xi_{3}$ are the principal strain rates and $\lambda$ is a nonnegative multiplier. Equations (15) and (16) are supplemented with the condition that the principal stress and strain rate directions coincide. In the case under consideration the equilibrium equations in the cylindrical coordinate system are

$$
\begin{aligned}
\frac{\partial \sigma}{\partial r}+\frac{\partial \tau_{r r}}{\partial r}+\frac{\partial \tau_{r z}}{\partial z}+\frac{\tau_{r r}-\tau_{\theta \theta}}{r} & =0 \\
\frac{\partial \tau_{r z}}{\partial r}+\frac{\partial \sigma}{\partial z}+\frac{\partial \tau_{z z}}{\partial z}+\frac{\tau_{r z}}{r} & =0
\end{aligned}
$$

where

$$
\begin{aligned}
\sigma & =\frac{\sigma_{1}+\sigma_{2}+\sigma_{3}}{3}, \\
\tau_{r r} & =\sigma_{r r}-\sigma, \\
\tau_{\theta \theta} & =\sigma_{\theta \theta}-\sigma, \\
\tau_{z z} & =\sigma_{z z}-\sigma, \\
\tau_{r z} & =\sigma_{r z} .
\end{aligned}
$$

It is reasonable to assume that

$$
\sigma_{2}=\sigma_{\theta \theta} .
$$

This assumption should be verified a posteriori. It follows from (15) and (16) that

$$
\tau_{f}=\frac{\sigma_{0}}{\sqrt[m]{1+2^{m-1}}}
$$

The basic assumption concerning the velocity field is [1]

$$
\frac{u_{r}}{U}=-\eta(\rho)
$$

where $\eta(\rho)$ is a function of $\rho$ and $\rho=r / b_{0}$. Using (21) the radial and circumferential strain rates are found as

$$
\begin{aligned}
& \xi_{r r}=-\frac{U}{b_{0}} \frac{d \eta}{d \rho}, \\
& \xi_{\theta \theta}=-\frac{U}{b_{0}} \frac{\eta}{\rho} .
\end{aligned}
$$

Then, the axial strain rate is determined from the incompressibility equations as

$$
\xi_{z z}=\frac{U}{b_{0} \rho} \frac{d(\eta \rho)}{d \rho} .
$$

Since $\xi_{z z}=\partial u_{z} / \partial z$ and the right hand side of (23) is independent of $z$, this equation can be immediately integrated to give

$$
\frac{u_{z}}{U}=\frac{\zeta}{\rho} \frac{d(\eta \rho)}{d \rho}+\mu(\rho)
$$

where $\zeta=z / b_{0}$ and $\mu(\rho)$ is an arbitrary function of its argument. It follows from (21) and (24) that the shear strain rate in the cylindrical coordinate system is

$$
\xi_{r z}=\frac{U}{2 b_{0}} \frac{d}{d \rho}\left[\frac{d(\eta \rho)}{\rho d \rho}\right] \zeta+\frac{U}{2 b_{0}} \frac{d \mu}{d \rho} .
$$

Let $\varphi$ be the inclination of the $\sigma_{1}$ principal stress direction to the $r$-axis, measured anticlockwise. Then,

$$
\tan 2 \varphi=\frac{2 \tau_{r z}}{\tau_{r r}-\tau_{z z}}=\frac{2 \xi_{r z}}{\xi_{r r}-\xi_{z z}} .
$$

It follows from (22), (25), and (26) that

$$
\tan 2 \varphi=-\frac{(d / d \rho)[d(\eta \rho) / \rho d \rho] \zeta+d \mu / d \rho}{[d \eta / d \rho+(1 / \rho)(d(\rho \eta) / d \rho)]}
$$

The solution derived in [1] suggests that $\varphi$ is independent of $z$. Then, it follows from (27) that $d(\eta \rho) / d \rho=2 C \rho$ where $C$ is constant. Integrating this equation gives

$$
\eta=C \rho+\frac{C_{1}}{\rho},
$$

where $C_{1}$ is constant of integration. It is seen from (21) that the boundary conditions (1) and (2) are equivalent to the conditions $\eta=1$ for $\rho=1$ and $\eta=0$ for $\rho=a$, respectively. Using these boundary conditions the constants $C$ and $C_{1}$ are determined from (28) as

$$
\begin{gathered}
C=\frac{1}{\left(1-a^{2}\right)}, \\
C_{1}=-\frac{a^{2}}{\left(1-a^{2}\right)} .
\end{gathered}
$$

Substituting (29) into (28) results in

$$
\eta=\frac{\left(\rho^{2}-a^{2}\right)}{\left(1-a^{2}\right) \rho} .
$$

Substituting (30) into (27) and using (26) result in

$$
\frac{2 \tau_{r z}}{\tau_{r r}-\tau_{z z}}=\frac{\rho^{2}\left(a^{2}-1\right)}{\left(a^{2}+3 \rho^{2}\right)} \frac{d \mu}{d \rho} .
$$


It is convenient to introduce the stress variables

$$
\begin{gathered}
s=\frac{\sigma_{1}+\sigma_{3}}{2 \sigma_{0}}, \\
t=\frac{\sigma_{1}-\sigma_{3}}{2 \sigma_{0}}, \\
s_{\theta \theta}=\frac{\sigma_{\theta \theta}}{\sigma_{0}} .
\end{gathered}
$$

It is evident from (14) that $t>0$. Using (32) it is possible to express the stress components in the cylindrical coordinate system as

$$
\begin{aligned}
& \frac{\sigma_{r r}}{\sigma_{0}}=s+t \cos 2 \varphi, \\
& \frac{\sigma_{z z}}{\sigma_{0}}=s-t \cos 2 \varphi, \\
& \frac{\sigma_{r z}}{\sigma_{0}}=t \sin 2 \varphi .
\end{aligned}
$$

The direction of flow requires that $\sigma_{r z}>0$ (Figure 3). Assume that $\sigma_{z z}>\sigma_{r r}$. This assumption should be verified a posteriori. Then, it is possible to find from (32) and (33) that

$$
\frac{\pi}{4} \leq \varphi \leq \frac{\pi}{2}
$$

Equation (19) dictates that $\xi_{\theta \theta}=\xi_{2}$. Therefore,

$$
\frac{\xi_{r r}-\xi_{z z}}{\xi_{\theta \theta}}=\frac{\left(\xi_{1}-\xi_{3}\right)}{\xi_{2}} \cos 2 \varphi
$$

Substituting (16), (22), and (28) into this equation and using (32) and (33) yield

$$
\begin{aligned}
& \frac{\left(3 \rho^{2}+a^{2}\right)}{\left(\rho^{2}-a^{2}\right)} \\
& =\frac{\left[\left(s+t-s_{\theta \theta}\right)^{m-1}+2(2 t)^{m-1}+\left(s_{\theta \theta}+t-s\right)^{m-1}\right] \cos 2 \varphi}{\left[\left(s_{\theta \theta}-s+t\right)^{m-1}-\left(s-s_{\theta \theta}+t\right)^{m-1}\right]} .
\end{aligned}
$$

It follows from (19) and (32) that the yield criterion (15) can be rewritten as

$$
\left[\frac{\left(s+t-s_{\theta \theta}\right)^{m}+\left(s_{\theta \theta}-s+t\right)^{m}+(2 t)^{m}}{2}\right]^{1 / m}=1 .
$$

Substituting (33) into the equilibrium equations (17) and assuming that $t$ is independent of $z$ yield

$$
\begin{aligned}
\frac{\partial s}{\partial \rho}+\frac{d(t \cos 2 \varphi)}{d \rho}+\frac{s-s_{\theta \theta}+t \cos 2 \varphi}{\rho} & =0, \\
\frac{\partial s}{\partial \zeta}+\frac{d(t \sin 2 \varphi)}{d \rho}+\frac{t \sin 2 \varphi}{\rho} & =0 .
\end{aligned}
$$

These equations have a solution if and only if

$$
\begin{aligned}
s & =C_{2}(\rho)+C_{3} \zeta, \\
s-s_{\theta \theta} & =\beta(\rho),
\end{aligned}
$$

where $C_{3}$ is constant and $C_{2}(\rho)$ and $\beta(\rho)$ are arbitrary functions of $\rho$. Substituting (39) into (38) results in

$$
\begin{aligned}
\frac{d\left(C_{2}+t \cos 2 \varphi\right)}{d \rho}+\frac{\beta(\rho)+t \cos 2 \varphi}{\rho} & =0, \\
\frac{d(t \sin 2 \varphi)}{d \rho}+\frac{t \sin 2 \varphi}{\rho} & =-C_{3} .
\end{aligned}
$$

Equation (40b) can be immediately integrated to give

$$
t \sin 2 \varphi=-\frac{C_{3} \rho}{2}+\frac{C_{4}}{\rho},
$$

where $C_{4}$ is constant of integration. Using (33) the boundary conditions (4) and (5) are transformed to $t \sin 2 \varphi=0$ for $\rho=$ 1 and $t \sin 2 \varphi=\tau_{f} / \sigma_{0}$ for $\rho=a$, respectively. These boundary conditions and (41) combine to give

$$
\begin{aligned}
& C_{3}=\frac{2 a}{\sqrt[m]{1+2^{m-1}}\left(1-a^{2}\right)}, \\
& C_{4}=\frac{a}{\sqrt[m]{1+2^{m-1}}\left(1-a^{2}\right)} .
\end{aligned}
$$

Therefore, (41) becomes

$$
t=\frac{1}{2}\left(\frac{1}{\rho}-\rho\right) \frac{C_{3}}{\sin 2 \varphi} .
$$

Eliminating $t$ and $s-s_{\theta \theta}$ in (36) and (37) by means of (39) and (43) yields

$$
\begin{aligned}
& \frac{\left(3 \rho^{2}+a^{2}\right)}{\left(\rho^{2}-a^{2}\right)}=\frac{\left\{\left[2 \beta \rho \sin 2 \varphi+C_{3}\left(1-\rho^{2}\right)\right]^{m-1}+2^{m} C_{3}^{m-1}\left(1-\rho^{2}\right)^{m-1}+\cdots+\left[C_{3}\left(1-\rho^{2}\right)-2 \beta \rho \sin 2 \varphi\right]^{m-1}\right\} \cos 2 \varphi}{\left[C_{3}\left(1-\rho^{2}\right)-2 \beta \rho \sin 2 \varphi\right]^{m-1}-\left[2 \beta \rho \sin 2 \varphi+C_{3}\left(1-\rho^{2}\right)\right]^{m-1}}, \\
& {\left[2 \beta \rho \sin 2 \varphi+C_{3}\left(1-\rho^{2}\right)\right]^{m}+\left[C_{3}\left(1-\rho^{2}\right)-2 \beta \rho \sin 2 \varphi\right]^{m}+\cdots+2^{m} C_{3}^{m}\left(1-\rho^{2}\right)^{m}=2^{m+1} \rho^{m} \sin ^{m} 2 \varphi .}
\end{aligned}
$$


Equations (44) should be solved for $\beta(\rho)$ and $\varphi(\rho)$ numerically. $C_{3}$ should be eliminated by means of (42). Once these equations have been solved, $t$ can be found from (42) and (43) and, then, $C_{2}$ is determined from (40a) as

$$
C_{2}=-t \cos 2 \varphi+\int_{a}^{\rho} \frac{(\beta+t \cos 2 \varphi)}{\chi} d \chi+C_{5}
$$

Here $C_{5}$ is constant of integration and $\chi$ is a dummy variable of integration. $\beta, t$, and $\varphi$ in the integrand are understood to be functions of $\chi$. The state of stress in the matrix is given by (33), (39), and (45). Then, $C_{5}$ and $C$ involved in (9) can be determined by means of the approach outlined in Section 2.

\section{Effect of the Yield Criterion of the Matrix on Brittle Fracture of the Fibre}

It follows from (33), (39), and (45) that

$$
\begin{aligned}
& \frac{\sigma_{r r}}{\sigma_{0}}=C_{3} \zeta+\int_{a}^{\rho} \frac{(\beta+t \cos 2 \varphi)}{\chi} d \chi+C_{5}, \\
& \frac{\sigma_{z z}}{\sigma_{0}}=C_{3} \zeta-2 t \cos 2 \varphi+\int_{a}^{\rho} \frac{(\beta+t \cos 2 \varphi)}{\chi} d \chi+C_{5} .
\end{aligned}
$$

These equations are valid in the range $L-L_{c} \leq z \leq L$ (Figure 3). Substituting (9) and (46) into (6) and (10) yields

$$
\begin{aligned}
C\left(\frac{l}{l_{c}}-1\right)+C_{5} \\
=\frac{C_{3}}{2}\left(l_{c}-2 l\right)-\int_{a}^{1} \frac{(\beta+t \cos 2 \varphi)}{\rho} d \rho, \\
C-C_{5} \\
=C_{3}\left(l-l_{c}\right)-1 \\
\quad+\frac{2}{\left(1-a^{2}\right)} \int_{a}^{1} \rho \int_{a}^{\rho} \frac{(\beta+t \cos 2 \varphi)}{\chi} d \chi d \rho \\
\quad-\frac{4}{\left(1-a^{2}\right)} \int_{a}^{1} \rho t \cos 2 \varphi d \rho,
\end{aligned}
$$

respectively. Here $l=L / b_{0}$ and $l_{c}=L_{c} / b_{0}$. Equations (47) and (48) constitute a linear system for $C$ and $C_{5}$. The solution to this system can be found with no difficulty. Once the value of $C_{5}$ has been found, $P$ and its dimensionless representation, $q$, are determined from (7) and (46) as

$$
\begin{aligned}
\frac{P}{\pi \sigma_{0} b_{0}^{2}}= & q \\
= & \left(C_{3} l+C_{5}\right)\left(1-a^{2}\right)-4 \int_{a}^{1} \rho t \cos 2 \varphi d \rho \\
& +2 \int_{a}^{1} \rho \int_{a}^{\rho} \frac{(\beta+t \cos 2 \varphi)}{\chi} d \chi d \rho .
\end{aligned}
$$

In order to evaluate the effect of the yield criterion on the tensile force at which the fibre breaks, it is convenient to introduce the parameter $\delta$ as

$$
\delta=\frac{\left|q-q_{s}\right|}{q_{S}} \cdot 100 \%
$$

Equations (44) have been solved numerically in the range $1.05 \leq m \leq 20$. Condition (19) has been verified using (32), (33), (39), and (45). Then, $q$ has been calculated by means of (42), (47), (48), and (49). It has been found by means of (11) and (50) that $\delta<1.5 \%$ for typical values of $a, l$, and $T / \sigma_{0}$ provided in [1]. Therefore, (11) derived in that paper is a very good approximation for the tensile load at which the fibre breaks independently of the yield criterion of the matrix.

\section{Conclusions}

Brittle fracture of fibres in continuous fibre reinforced composites subjected to tensile loading in the direction parallel to the fibres has been predicted by means of the theory developed in [1]. A distinguished feature of this new solution is that the generalized yield criterion proposed in [15] has been adopted for the matrix. The solution has been compared to the analytic solution for Tresca's yield criterion derived in [1]. It has been found that the magnitude of the tensile load at which the fibre breaks is practically independent of the yield criterion of the matrix. In particular, formula (11) obtained in [1] predicts this magnitude for any isotropic pressureindependent yield criterion with a very high accuracy. In general, this feature of the solution is capable of experimental verification and hence provides a means for testing the theory. However, such verification requires the determination of the yield criterion of the matrix before testing the composite. To the best of authors' knowledge, no research that includes both testing the matrix material and testing the composite has been reported in the literature. Therefore, the present paper may be considered as an encouragement to experimentalists to attempt to verify the theoretical predictions made.

A new result of academic interest is the solution for the constitutive equations (15) and (16) supplemented with the equilibrium equations and satisfying the boundary conditions (1)-(5). This solution can be regarded as a generalization of the famous Prandtl's solution for plane strain compression of a plastic layer between parallel, rough plates (see, e.g., [23]). In contrast to the plane strain problem, the solution to the axisymmetric problem depends on the yield criterion chosen. A distinguished feature of the present solution is that the generalized yield criterion has been adopted.

\section{Competing Interests}

The authors declare that they have no competing interests.

\section{Acknowledgments}

The reported study was funded by RFBR according to the research project no. 16-58-52051. 


\section{References}

[1] A. J. M. Spencer, "A theory of the failure of ductile materials reinforced by elastic fibres," International Journal of Mechanical Sciences, vol. 7, no. 3, pp. 197-209, 1965.

[2] G. J. Dvorak and Y. A. Bahei-El-Din, "Plasticity analysis of fibrous composites," Journal of Applied Mechanics, vol. 49, no. 2, pp. 327-335, 1982.

[3] C. Cheng and N. Aravas, "Creep of metal-matrix composites with elastic fibers-part I: continuous aligned fibers," International Journal of Solids and Structures, vol. 34, no. 31-32, pp. 4147-4171, 1997.

[4] H. Naji, S. M. Zebarjad, and S. A. Sajjadi, "The effects of volume percent and aspect ratio of carbon fiber on fracture toughness of reinforced aluminum matrix composites," Materials Science and Engineering A, vol. 486, no. 1-2, pp. 413-420, 2008.

[5] I. T. Lee, Y. Q. Wang, Y. Ochi, S. I. Bae, K. S. Han, and J. I. Song, "Effect of short fiber reinforcement on the fracture toughness of metal matrix composites," Advanced Composite Materials, vol. 19, no. 1, pp. 41-53, 2010.

[6] S. Ghosh, Y. Ling, B. Majumdar, and R. Kim, "Interfacial debonding analysis in multiple fiber reinforced composites," Mechanics of Materials, vol. 32, no. 10, pp. 561-591, 2000.

[7] A. Caporale, R. Luciano, and E. Sacco, "Micromechanical analysis of interfacial debonding in unidirectional fiber-reinforced composites," Computers \& Structures, vol. 84, no. 31-32, pp. 2200-2211, 2006

[8] S. Li and S. Ghosh, "Modeling interfacial debonding and matrix cracking in fiber reinforced composites by the extended voronoi cell FEM," Finite Elements in Analysis and Design, vol. 43, no. 5, pp. 397-410, 2007.

[9] D. H. Allen, R. H. Jones, and J. G. Boyd, "Micromechanical analysis of a continuous fiber metal matrix composite including the effects of matrix viscoplasticity and evolving damage," Journal of the Mechanics and Physics of Solids, vol. 42, no. 3, pp. 505-529, 1994.

[10] C. R. Ananth, S. R. Voleti, and N. Chandra, "Effect of fiber fracture and interfacial debonding on the evolution of damage in metal matrix composites," Composites Part A: Applied Science and Manufacturing, vol. 29, no. 9-10, pp. 1203-1211, 1998.

[11] Z. P. Luo and C. Y. Sun, "Effect of the interfacial bonding status on the tensile fracture characteristics of a boron-fiberreinforced aluminum composite," Materials Characterization, vol. 50, no. 1, pp. 51-58, 2003.

[12] S. Fukumoto, A. Hirose, and K. F. Kobayashi, "Evaluation of the strength of diffusion bonded joints in continuous fiber reinforced metal matrix composites," Journal of Materials Processing Technology, vol. 68, no. 2, pp. 184-191, 1997.

[13] T. Pacheco, H. Nayeb-Hashemi, and H. E. M. Sallam, "The effects of matrix and fiber properties on the mechanical behavior and acoustic emission in continuous fiber reinforced metal matrix composites," Materials Science and Engineering A, vol. 247, no. 1-2, pp. 88-96, 1998.

[14] J. L. Bucaille, A. Rossoll, B. Moser, S. Stauss, and J. Michler, "Determination of the matrix in situ flow stress of a continuous fibre reinforced metal matrix composite using instrumented indentation," Materials Science and Engineering A, vol. 369, no. 1-2, pp. 82-92, 2004.

[15] W. F. Hosford, "A generalized Isotropic Yield Criterion," International Journal of Applied Mechanics, vol. 39, no. 2, pp. 607-609, 1972.
[16] W. H. Yang, "A generalized von Mises criterion for yield and fracture," Journal of Applied Mechanics, vol. 47, no. 2, pp. 297300, 1980.

[17] S. R. Voleti, C. R. Ananth, and N. Chandra, "Effect of fiber fracture and matrix yielding on load sharing in continuous fiber metal matrix composites," Journal of Composites Technology o Research, vol. 20, no. 4, pp. 203-209, 1998.

[18] R. T. Shield, "Plastic flow in a converging conical channel," Journal of the Mechanics and Physics of Solids, vol. 3, no. 4, pp. 246-258, 1955.

[19] S. Alexandrov and F. Barlat, "Modeling axisymmetric flow through a converging channel with an arbitrary yield condition," Acta Mechanica, vol. 133, no. 1-4, pp. 57-68, 1999.

[20] S. E. Aleksandrov and R. V. Goldstein, "Generalization of the prandtl solution to the case of axisymmetric deformation of materials obeying the double shear model," Mechanics of Solids, vol. 47, no. 6, pp. 654-664, 2012.

[21] S. Alexandrov and Y.-R. Jeng, "A generalization of Prandtl's and Spencer's solutions on axisymmetric viscous flow," Archive of Applied Mechanics, vol. 81, no. 4, pp. 437-449, 2011.

[22] A. J. M. Spencer, "Deformation of ideal granular materials," in Mechanics of Solids: The Rodney Hill 60th Anniversary Volume, H. G. Hopkins and M. J. Sewell, Eds., pp. 607-652, Pergamon Press, Oxford, UK, 1982.

[23] R. Hill, The Mathematical Theory of Plasticity, Clarendon Press, Oxford, UK, 1950. 

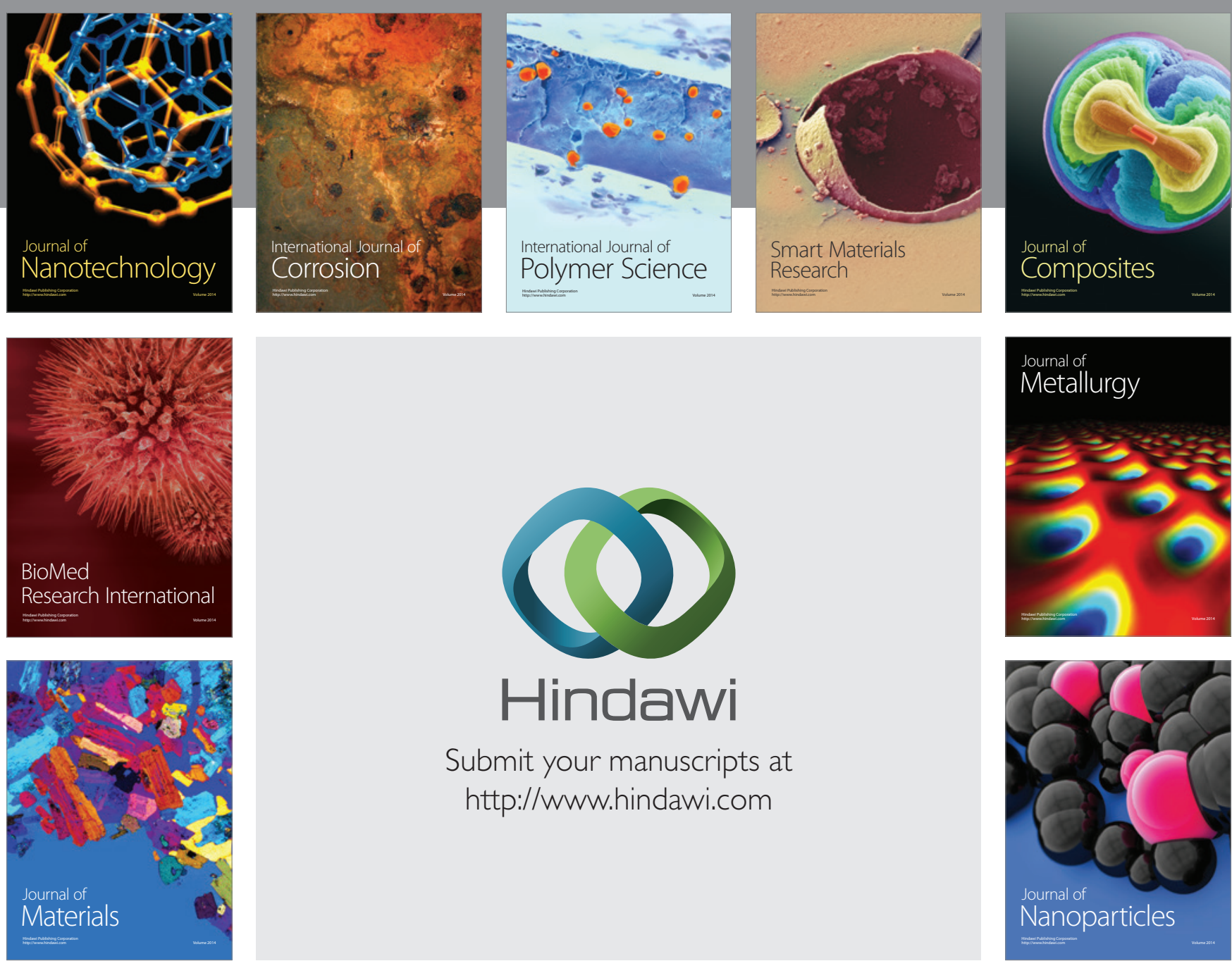

\section{Hindawi}

Submit your manuscripts at

http://www.hindawi.com

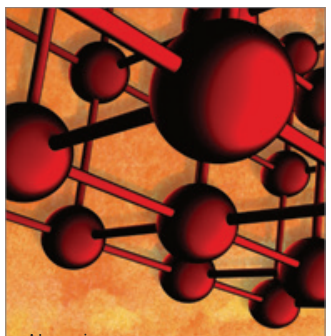

Materials Science and Engineering
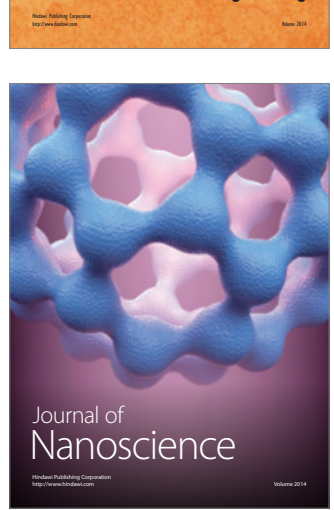
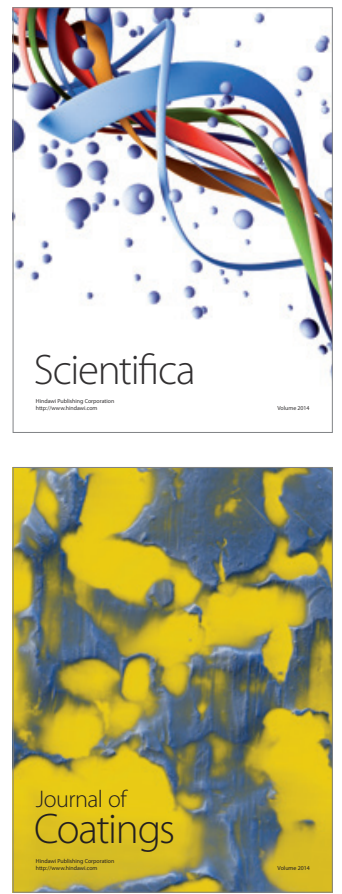
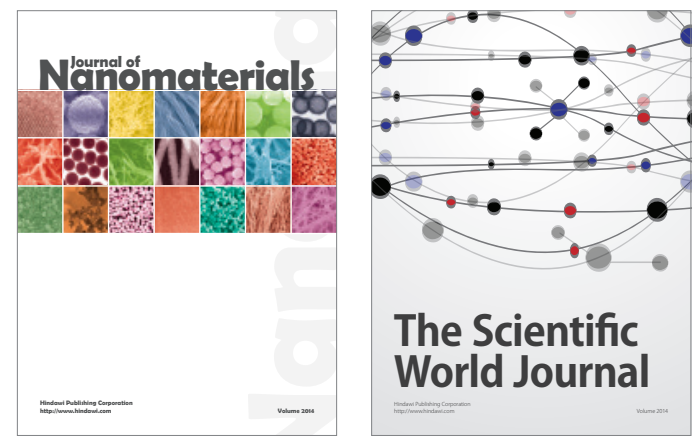

The Scientific World Journal
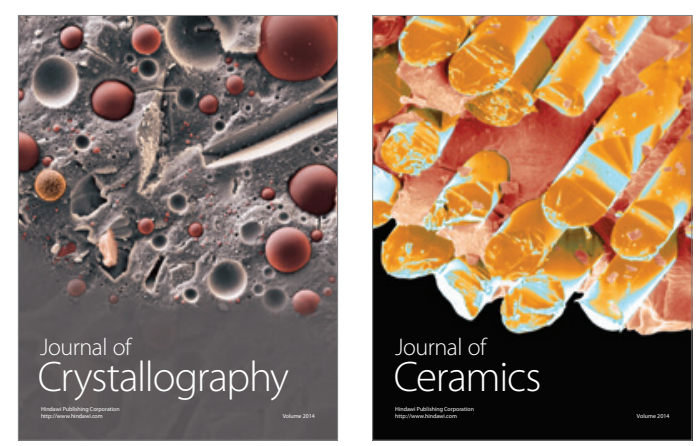
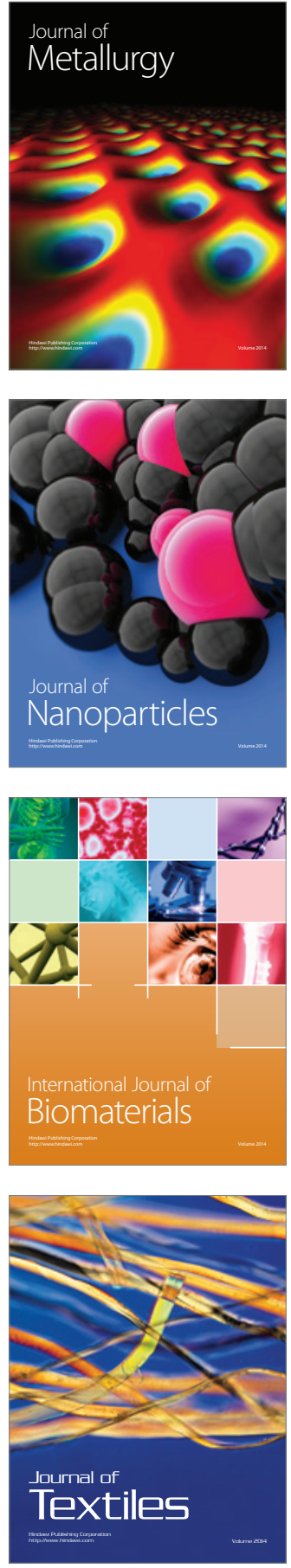